\title{
Knockout of Low Molecular Weight FGF2 Attenuates Atherosclerosis by Reducing Macrophage Infiltration and Oxidative Stress in Mice
}

\author{
Wenjing Lianga,d Qian Wang ${ }^{c}$ Hui Ma ${ }^{b}$ Wenjiang Yan ${ }^{a}$ Jingjing Yang ${ }^{a}$ \\ aThe Key Laboratory of Cardiovascular Remodeling and Function Research, Chinese Ministry of \\ Education and Chinese Ministry of Health, and The State and Shandong Province Joint Key Laboratory \\ of Translational Cardiovascular Medicine, Qilu Hospital of Shandong University, Jinan, Shandong, \\ 'Shandong Provincial Hospital, Affiliated with Shandong University, Jinan, Shandong, 'Department of \\ Cardiology, Shandong Province Hospital affiliated to Shandong University, Jinan, Shandong, China, \\ dDepartment of Pharmacology, University of California, San Diego, La Jolla, CA, USA
}

\section{Key Words}

Atherosclerosis • 18-kDa FGF-2 Isoform • Macrophage • NADPH

\begin{abstract}
Background/Aims: Fibroblast growth factor 2 (FGF2) plays a predominant role during angiogenesis in the adventitia and in atherosclerotic plaque. A dilemma exists, however, as to whether angiogenic stimulation by FGF2 for the prevention and treatment of atherogenesis is feasible. The aim of this study is to investigate the effect of the 18-kDa FGF-2 isoform on atherosclerosis progression in high-fat diet-fed apolipoprotein E knockout (ApoE-/-) mice. Methods: We established a model of atherosclerosis using ApoE and 18-kDa FGF-2 gene double knockout mice. They were randomly divided into three groups depending on the duration of diet: 8 weeks, 12 weeks and 16 weeks. Then, we studied the morphology and inflammatory factor staining in the atherosclerosis plaque of these mice. Results: Knockout of the 18-kDa FGF-2 isoform did not change the metabolic characteristics of the mice. Compared to the control group, knockout of the 18-kDa FGF-2 isoform significantly attenuated atherogenesis, reduced aortic plaques, reduced macrophage infiltration and suppressed oxidative stress in mice fed with a high fat diet at all-time points. Conclusions: 18-kDa FGF-2 aggravated the inflammatory reaction of atherosclerosis.

\section{Introduction}

Atherosclerosis is a chronic inflammatory disease of the vascular wall. It is a complex process involving a number of inflammatory cells and cytokine interactions throughout 
different stages of its development. It is caused by circulating low density lipoprotein (LDL) which enters the sub-endothelial space of the blood vessel [1, 2]. Once LDL is oxidized by reactive oxygen species (ROS), adhesion molecules such as vascular endothelial cell adhesion molecule-1 (VCAM-1), intercellular adhesion molecule-1 (ICAM-1), E-selectin and P-selectin are up-regulated. Additionally, expression of chemokines, such as monocyte chemoattractant protein-1 (MCP-1) is increased in endothelial cells [3, 4]. Up-regulation of adhesion molecules leads to the recruitment of monocytes and T-lymphocytes to the vessel wall, which is a key factor in maintaining the inflammatory process [4-6]. The most abundant immune cell type in atherosclerotic lesions is the macrophage, which is involved in all pathological stages of atherosclerosis [7, 8]. Macrophages secrete chemokines such as MCP-1, and produce angiogenic factors such as basic fibroblast growth factor (bFGF), also known as FGF2 or FGF- $\beta$, a well-characterized angiogenic growth factor. FGF2 then promotes the growth of microvessels within the plaque to leasing to neoplasia, hemorrhage and thrombosis.

Angiogenic stimulation by FGF2 has been widely regarded as a promising strategy for treating patients with arteriosclerotic coronary artery disease (CAD) through protection of the arterial endothelium $[9,10]$. This strategy aims to improve cardiac function and reduce the risk of myocardial infarction by improving myocardial blood supply $[11,12]$. Conversely, angiogenesis may contribute to the growth of atherosclerotic lesions and play a key role in the destabilization of plaque, leading to rupture $[13,14]$. Clinical trials thus far cannot provide significant evidence for the efficacy of FGF2, and in view of unavoidable side effects occurring with FGF2 treatment, its clinical application is still controversial. Thus, the role of angiogenesis in atherosclerosis is endlessly debated, and further work is necessary.

FGF2 consists of multiple protein isoforms, including low molecular weight (LMW), and high molecular weight (HMW) produced by alternative translation from the FGF2 gene $[15,16]$. Due to differential functions that LMW and HMW isoforms contribute towards in the cardiovascular system, significant efforts have been made to determine their individual characteristics in vivo and ex vivo using experiments in mammals. The LMW, 18-kDa FGF2 isoform, is translated from a conventional AUG start codon and consists of 155 amino acids, representing the core sequence common to all FGF2 isoforms [15]. At present, FGF2associated research mainly focuses on functional studies of the 18-kDa FGF-2 isoform. In our study, we constructed a ApoE and 18-kDA-FGF2 gene double knockout mouse for exploring the underlying role of the $18-\mathrm{kDa}$ FGF2 isoform in the progression of atherosclerosis during in different time points after being fed with a high-fat diet.

\section{Materials and Methods}

\section{Animal model}

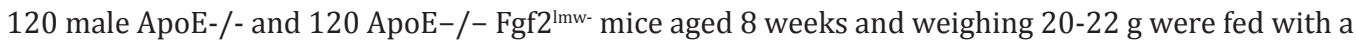
high fat diet during the entire experimental period. 18-kDa-FGF2 knockout (Fgf2 ${ }^{\text {Imw- }}$ ) mice were purchased from Jackson Laboratory (JAX Stock No. 010698).

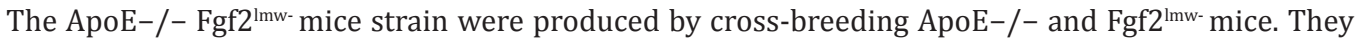
were backcrossed for a minimum of 20 generations. The genotyping results has been showed in Fig. 1. All animals were conventionally housed and kept on a $12 \mathrm{~h}$ light/12 h dark cycle with food and water freely available. The food composition of the high fat diet was $15 \%$ cocoa butter and $0.25 \%$ cholesterol. In order to investigate whether the effect of 18-kDa FGF2 knockout on atherosclerosis is dependent on the stage of the disease process, mice were randomly divided into three groups depending on the duration of diet: 8 weeks, 12 weeks and 16 weeks. Then, we studied the plaque morphology of these mice. All of the animal care and experimental procedures were in accordance with the Animal Management Rule of the Chinese Ministry of Health (Documentation 55, 2001), and were approved by the Animal Care and Use Committee of Shandong University. 


\section{Cellular Physiology Cell Physiol Biochem 2018;45:1434-1443 \begin{tabular}{l|l|l} 
and Biochemistry & $\begin{array}{l}\text { DOI: 10.1159/000487569 } \\
\text { Published online: February 21, } 2018\end{array}$ & $\begin{array}{l}\text { () } 2018 \text { The Author(s). Published by S. Karger AG, Basel } \\
\text { ww.karger.com/cpb }\end{array}$ \\
\hline
\end{tabular}}

Liang et al.: Role of Low Molecular Weight FGF2 in Atherosclerosis

\section{Measurement of plasma components}

Mice were euthanized and blood was collected through cardiac puncture. Serum levels of total cholesterol (TC), triglyceride (TG), low-density lipoprotein-cholesterol (LDL-C) and high-density lipoprotein-cholesterol (HDL-C) were measured, using a biochemistry automatic analyzer (HITACHI 7170A, Hitachi, Tokyo, Japan).

\section{Atherosclerotic lesion analysis}

The heart and aortic tissue were removed from the ascending aorta to the ileal bifurcation and placed in 4\% paraformaldehyde for at least 16 hours. After fixation, the adventitia was thoroughly cleaned under a dissecting microscope. For analyzing the lesion area in the aortic root, the heart with attached aortic arch was embedded in OCT and sectioned at $5 \mu \mathrm{m}$ using a Leica cryostat for histopathology and immunohistochemistry staining. Four consecutive sections were collected from each mouse and stained with Oil Red 0 for neutral lipids, and counterstained with hematoxylin to visualize the nuclei. Images of plaques were captured with an Olympus microscope connected to a QImaging Retiga CCD camera. The aortic lesion size of each animal was obtained by the averaging of lesion areas in four sections from the same mouse. For analysis of the lesion area in the aortic arch, the intimal surface was exposed by a longitudinal cut from the ascending arch to $5 \mathrm{~mm}$ distal of the left subclavian artery to allow the lumen of the aortic arch to be laid flat.

\section{Histopathology and immunohistochemistry}

All immunohistochemical staining (IHC) was detected by DAB. IHC was used to detect target protein expression. Rehydrated sections obtained from the thoracic aorta and cryosections from the aortic root $(5 \mu \mathrm{m})$ were microwaved in citrate buffer for antigen retrieval. Sections were incubated in endogenous peroxidase (DAKO) and protein block buffer, and then with primary antibodies overnight at $4^{\circ} \mathrm{C}$ as indicated below. Slides were rinsed with PBS and incubated with secondary antibodies.

The primary antibody against macrophage-specific antigen (MOMA-2, diluted 1:150; ab33451, Abcam), vascular cell adhesion molecule-1 (VCAM-1, ab171123, diluted 1:200; Abcam), Nox4 (ab109225, diluted 1:200, Abcam), p47phox (sc-17844, diluted 1:200, Santa Cruz) and MCP-1 (ab7202, diluted 1:100, Abcam) were used for immunohistochemical staining. The contents and distribution were evaluated by analyzing positive staining areas using the computer-assisted morphometric analysis system Image-Pro Plus 6.0 (Media Cybernetics, Bethesda, MD, USA). The extent of atherosclerotic lesions in the entire aorta was measured by the positive oil red 0 staining area relative to the entire aorta en face.

Western blot

Total proteins were extracted from aortic tissues of mice. The protein level change of Fgf $2^{\text {lmw- }}$ was quantitatively analyzed using western blotting.

\section{Statistical analysis}

SPSS 13.0 for Windows (SPSS, Inc., Chicago, IL) was used for all data analysis. Continuous variables were expressed as mean \pm standard deviation (SD). Unpaired t-test was used for comparisons between two groups. Values of $P<0.05$ were considered statistically significant.

\section{Results}

ApoE-/- Fgf2 ${ }^{\text {lmw- }}$ double knockout mouse model building and genotyping analysis

The genotypes of mice were analyzed by agarose gel electrophoresis. Tag and Exchange

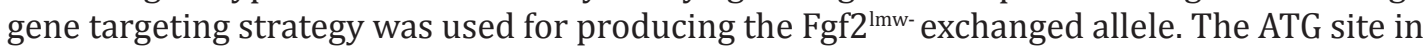
the genomic DNA was mutated, which also resulted in a diagnostic PstI site to divide the DNA into $476 \mathrm{bp}$ and a $90 \mathrm{bp}$ sections.

Genotyping analysis of ApoE-/- Fgf2 ${ }^{\text {lmw- }}$ mice showed a band at 265 bp (Apoe - / -) and bands at $476 \mathrm{bp}+90 \mathrm{bp}\left(\mathrm{Fgf}^{\mathrm{lmw}-/}\right)$; $90 \mathrm{bp}$ is too small to observe, so only a $476 \mathrm{bp}$ band was observed (Fig. 1). Western blot showed the complete absence of the 18 kDa-FGF2 (Fig. 1). 
Fig. 1. ApoE-/- Fgf $2^{\text {Imw- } /-m o u s e ~ b u i l d i n g ~}$ and genotype analysis; protein expression. (A) $2 \%$ agarose gel electrophoresis ApoE-/in a band at $265 \mathrm{bp}$, ApoE -/+ mice at $265 \mathrm{bp}$ and $155 \mathrm{bp}$ at each side of a belt, wild-type mice in a band at $155 \mathrm{bp}$. (B) $2 \%$ agarose gel electrophoresis showed that FGF2 -/- in a band at $476 \mathrm{bp}, \mathrm{FGF} 2-/+$ mice at $566 \mathrm{bp}$ and $476 \mathrm{bp}$ at each side of a belt. Wild-type mice in a band at $566 \mathrm{bp}$. Homozygous mice were completely cut into $476 \mathrm{bp}+90 \mathrm{bp} ; 90 \mathrm{bp}$ is too small to show up in the gel, so we only

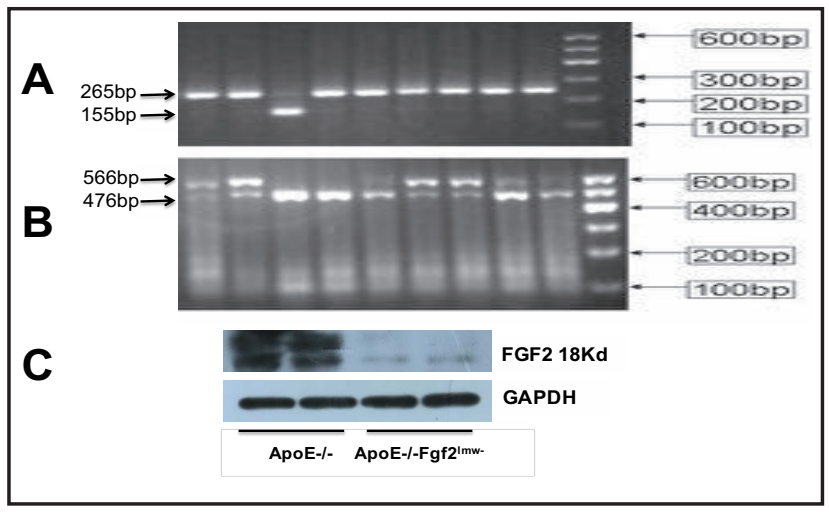
observed $566 \mathrm{bp}, 476 \mathrm{bp}$ bands to tell the appropriate genotype. (C) Representative protein expression of the low molecular weight isoform of FGF2.

Body weight and serum lipid profile

At the end of the experiment, there were no significant differences in metabolic parameters including total cholesterol, triglyceride, very-lowdensity lipoprotein (VLDL) cholesterol, LDL cholesterol, high-density lipoprotein (HDL) cholesterol, and glucose levels between ApoE-/- Fgf2 ${ }^{\text {lmw- }}$ and ApoE-/- mice (Table 1).

Low molecular weight isoform of FGF2 induced aortic lesions

The relative en face lesion area and the relative cross-sectional lesion area of the aorta were significantly decreased in the ApoE-/Fgf2 ${ }^{\text {lmw- }}$ mice compared to control ApoE-/- mice. Analysis of the 8-week HFD group showed the en face lesion area ratio was $17.2 \%$ and $9.8 \%$ in ApoE-/- mice and DKO mice, respectively. Histological cross-sections stained with Oil Red 0 of atherosclerotic lesions around the aortic sinus, indicating lipid content, were $23.3 \%$ in ApoE-/- mice and $9.4 \%$ in DKO mice. In the 12 -
Table 1. Body weight, blood glucose and serum lipid levels. TC: total cholesterol; TG: triglyceride; HDL-C: high-density lipoprotein cholesterol; LDL-C: low density lipoprotein cholesterol. There is no difference between the two groups among these indexes

\begin{tabular}{lcccccc}
\hline Groups & Body Weight (g) & $\begin{array}{c}\text { Glucose } \\
(\mathrm{mmol} / \mathrm{L})\end{array}$ & $\begin{array}{c}\mathrm{TC} \\
(\mathrm{mmol} / \mathrm{L})\end{array}$ & $\begin{array}{c}\text { TG } \\
(\mathrm{mmol} / \mathrm{L})\end{array}$ & $\begin{array}{c}\mathrm{HDL}-\mathrm{C} \\
(\mathrm{mmol} / \mathrm{L})\end{array}$ & $\begin{array}{c}\text { LDL-C } \\
(\mathrm{mmol} / \mathrm{L})\end{array}$ \\
\hline $\begin{array}{l}\text { ApoE-/- } \\
\text { (8-week HFD) }\end{array}$ & $29.3 \pm 0.3$ & $10.1 \pm 1.2$ & $21.2 \pm 5.3$ & $0.52 \pm 0.1$ & $1.4 \pm 0.3$ & $2.8 \pm 0.9$ \\
$\begin{array}{l}\text { DKO } \\
\text { (8-week HFD) }\end{array}$ & $28.8 \pm 1.2$ & $8.7 \pm 1.4$ & $21.3 \pm 3.8$ & $0.48 \pm 0.1$ & $1.5 \pm 0.3$ & $2.6 \pm 0.8$ \\
\hline
\end{tabular}

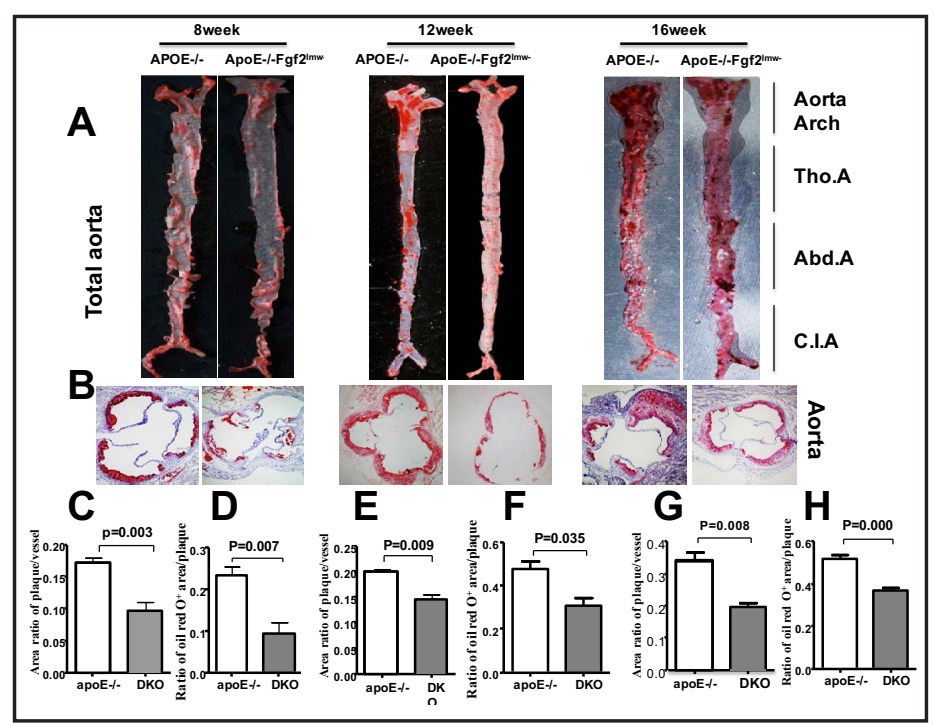

Fig. 2. Effects of knockout 18 -kDa FGF2 on aortic atherosclerotic plaque growth with 8,12 , and 16 weeks high fat diet treatment. After 8,12 , and 16 weeks of high fat diet treatment, all mice were sacrificed under anesthesia. (A) Whole aortas including thoracic and abdominal aortas showing atherosclerotic lesion analysis by Oil Red 0 staining. (B) Oil Red O staining on the aortic root section. (C, E, G) Quantitative data of atherosclerotic lesions in the whole aortas in 8, 12, and 16week HFD groups. (D, F, H) Quantitative analysis of atherosclerotic lesion size in the aortic root by Oil Red 0 staining in 8,12 , and 16week HFD groups (original magnification X100). N equals 5-8 in each group. 
Fig. 3. Macrophage marker expression in atherosclerotic lesions of aortic roots at 8 , 12 and 16 weeks of high fat diet treatment. (A) Immunohistochemical staining for macrophages (MOMA2) of the aortic lesions. (B, C, D) Quantitative analysis of MOMA-2 expression were measured in 8, 12 and 16 weeks high fat diet-fed groups (original magnification $\mathrm{X} 100$ ). $\mathrm{N}$ is $5-8$ in each group.

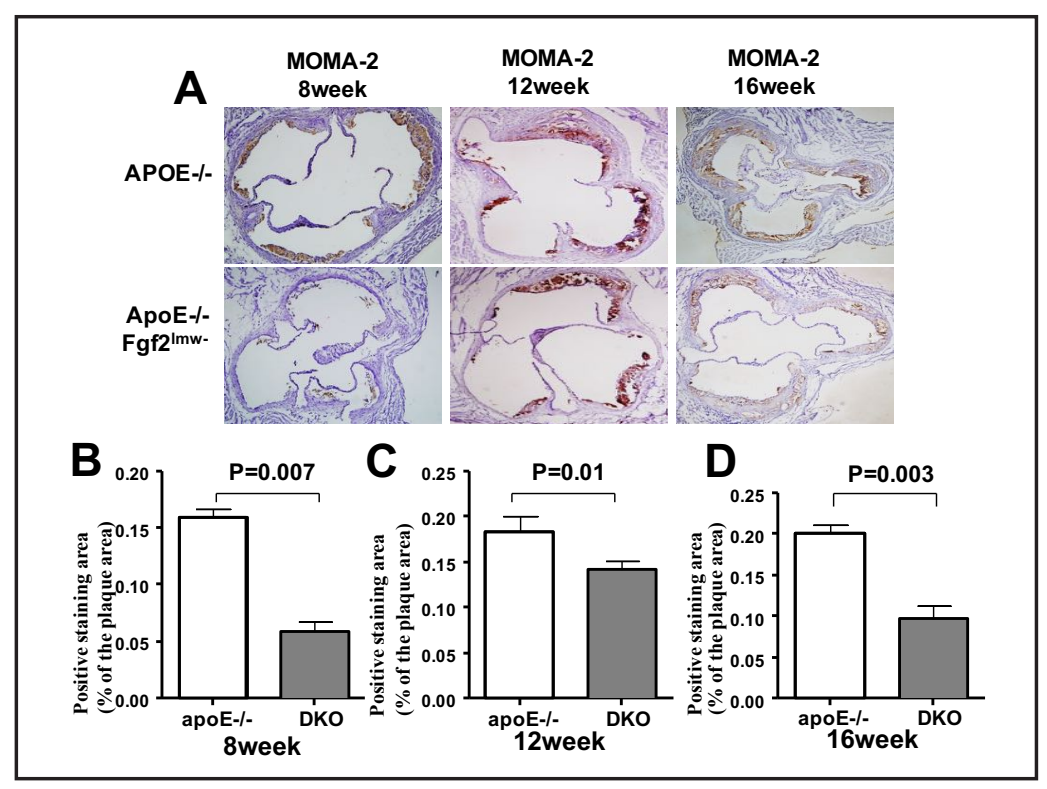

Fig. 4. MCP-1 expression in the root of the aorta atherosclerotic plaques at 8,12 and 16 weeks of high fat diet treatment. (A) Immunohistochemical staining for MCP-1 of the aortic lesions. (B C D) Quantitative analysis of MCP-1 expression in the two groups of mouse aorta. Data from 8, 12 and 16 weeks were measured separately (original magnification X100). $\mathrm{N}$ is $5-8$ in each group.

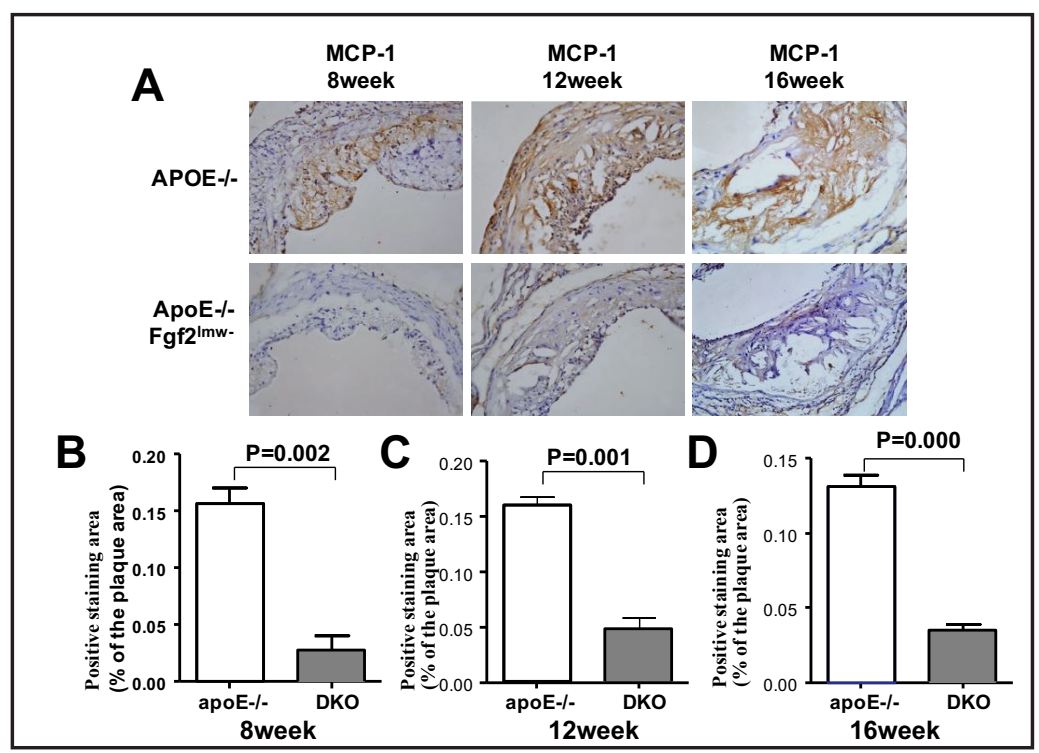

week HFD group, en face lesion areas were $20.0 \%$ and $14.6 \%$, and the aortic root lipid stained positive area ratios were $47.4 \%$ and $30.6 \%$ in ApoE-/- mice and DKO mice, respectively. The 16-week HFD group had the same trend, en face lesion area ratios were $34.0 \%$ and $19.6 \%$, and aortic root lipids contents ratios were 51.8\% and 36.7\% in ApoE-/- mice and DKO mice, respectively (Fig. 2). These results suggest that $18 \mathrm{kDa}-\mathrm{FGF} 2$ is involved in the development of atherosclerosis processing from early to advanced stages.

\section{Knockout of 18 kDa-FGF2 reduced macrophage infiltration}

To further reveal the role of $18 \mathrm{kDa}$-FGF2 in atherosclerosis plaque, we measured macrophages expression on the atherosclerotic lesions by staining with an antibody against the macrophage marker MOMA-2. The relative macrophage infiltration in aortic plaques as shown by IHC staining was significantly decreased: the positive staining area ratio was $16.0 \%$ in ApoE-/- mice compared to 5.9\% in DKO mice in the 8-week HFD group, 18.3\% compared to $14.2 \%$ in the 12 -week HFD group and $19.5 \%$ compared to $9.0 \%$ in the 16 week HFD group. This suggests that $18-\mathrm{kDa}$ FGF2 is involved with macrophage infiltration in atherosclerotic plaque (Fig. 3). 
Fig. 5. VCAM expression in aortic plaque at 8,12 and 16 weeks high fat diet treatment. (A) Immunohistochemical staining for VCAM-1 of the aortic lesions. (B C D) Quantitative analysis of VCAM-1 expression detected in mouse aorta at 8, 12 and 16 weeks high fat diet treatment (original magnification $\mathrm{X} 100$ ). $\mathrm{N}$ is $5-8$ in each group.

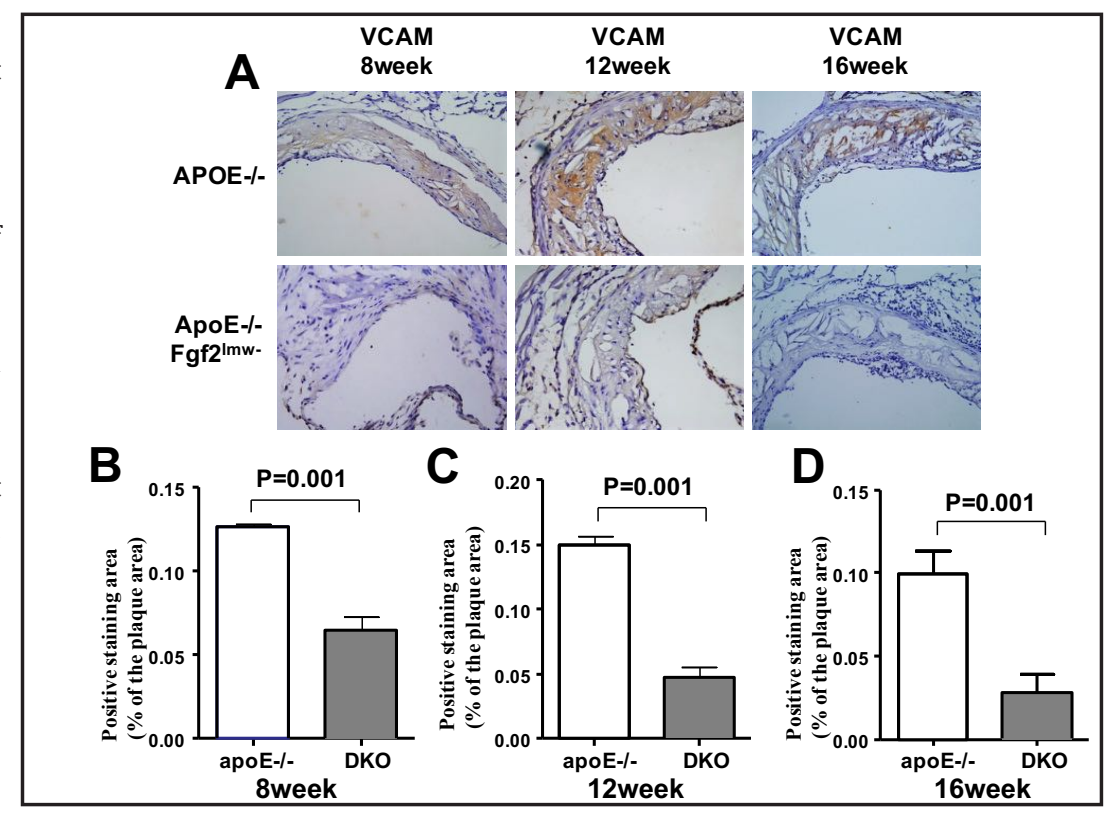

Fig. 6. NOX4 expression in aorta atherosclerosis plaque in different stages of the disease process. (A) Immunohistochemical staining for NOX4 expression in the aortic lesions. (B, C, D) Quantitative analysis of NOX4 expression of 8,12 and 16 weeks high fat diet treatment in control and DKO mice (original magnification $\mathrm{X} 100)$. $\mathrm{N}$ is 5-8 in each group.

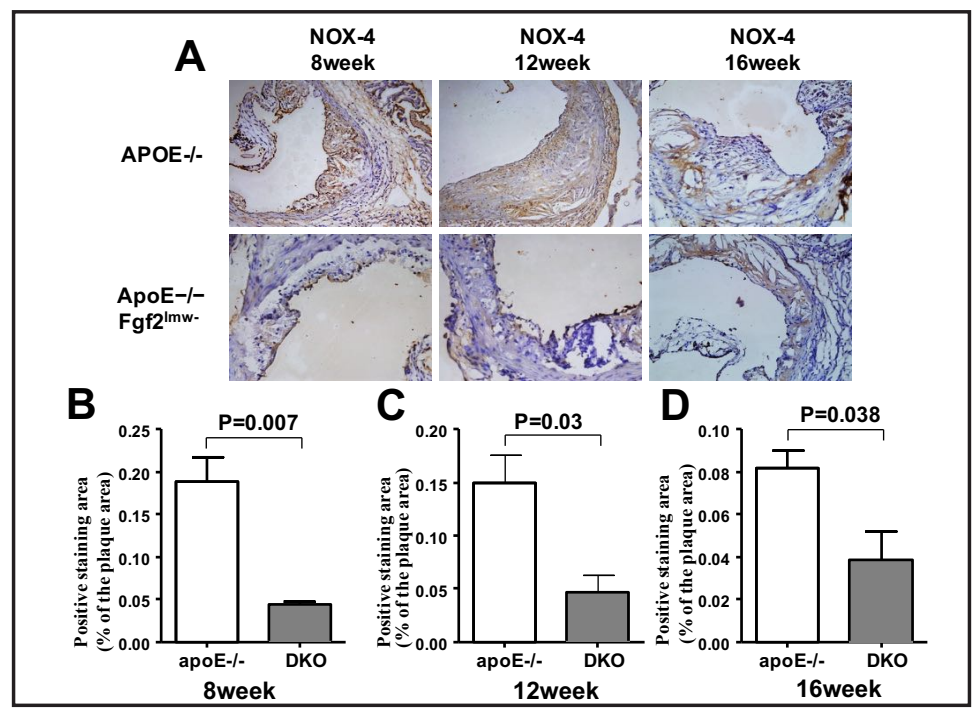

18-kDa FGF2 involved in chemokine and adhesion molecule expression

In atherosclerotic lesions, FGF2 and its receptors contribute to inflammatory processes and intimal thickening [17]. High expression of MCP-1 was detected in macrophage-rich atherosclerotic lesions in human and rabbit [18]. VCAM-1 has an increased expression at lesion-prone sites and has been involved in monocyte migration in atherosclerosis [19]. Prevention effect of 18-kDa FGF2 on the expression of MCP-1 and VCAM-1 in atherosclerotic plaques was evaluated in order to examine the proinflammatory, cytokine-stimulated mechanism involved in monocyte-endothelial adhesion. Results indicated that 18-kDa FGF2 isoform knockout markedly reduced MCP-1 expression. The positive staining area in ApoE/- mice was $15.6 \%$, whereas the DKO mice only had 2.8\% with the 8-week HFD group. In the 12 -week group, the ratio was $16.0 \%$ and $4.9 \%$ in control mice and DKO mice, respectively. This decreased from $12.2 \%$ to $3.0 \%$ in the 16 -week HFD group. The expression of VCAM-1 was also reduced in DKO mice in all three durations of HFD treatment. The positive staining ratio in DKO mice was 6.4\%, 4.8\% and 2.8\% in 8-week, 12 -week and 16-week, respectively, compared to the control ApoE-/- mice, which were $12.7 \%, 14.9 \%$ and $9.6 \%$ at the respective time-points. (Fig. 4 and 5).

\section{KARGER}


Knockout of 18-
$k D a$ FGF2 inhibited
NADPH oxidase
in atherosclerotic
lesions
NOX4, which is present in all vascular wall cells, is the most abundant NADPH oxidase (NOX) enzyme in the NOX family. It is involved in several initiating events in the process of atherosclerosis. Elevated reactive oxygen species has the ability to cause oxidative stress, and trigger endothelial dysfunction, platelet aggregation, and macrophage polarization [20]. Another NADPH oxidase cytosolic protein, $\mathrm{p} 47 \mathrm{phox}$ is involved in the atherosclerosis process via regulating NOX2 activation [21]. To test the correlation of expression of NADPH oxidase subunits and 18-kDa FGF2, we performed functional verification in vivo. We tested Nox 4 and p47phox expression in the atherosclerotic lesions and found that, compared to the ApoE-/- mice, the DKO mice possessed lower Nox4 and p47phox expression among the three different time-points (8week, 12-week and 16-week of HFD fed). The ratios of Nox4 positive staining in control ApoE-/- mice were $18.9 \%, 14.9 \%$ and $8.3 \%$, whereas all of them decreased to $4.3 \%, 4.7 \%$ and 3.9\% in DKO mice, respectively (Fig. 6). Similar results occurred with p47phox expression detection. The results showed that in control ApoE-/- mice, the ratios were 14.3\%, 14.0\% and $12.6 \%$ in 8-week, 12-week and 16-week HFD fed groups, however at all time-points the ratios reduced to $4.3 \%, 6.4 \%$ and $4.5 \%$ in DKO mice, respectively (Fig. 7).

\section{Discussion}

Our study provided evidence that knockout of low molecular weight FGF2 plays an important role in preventing the development of atherosclerosis. We conducted a series of experiments to prove that $18-\mathrm{kDa}$ FGF2 is involved in macrophage infiltration, the expression of chemokine and adhesion molecules, as well as expression of NADPH oxidase subunits in atherosclerotic lesions with different durations of high fat-fed double knockout mice.

FGF2 is involved in regulating numerous cellular functions in multiple cell types, including cell proliferation, differentiation, viability, adhesion and migration $[22,23]$. Function of FGF2 in cardiovascular disease also includes angiogenesis and blood vessel remodeling; it is widely considered as the most common angiogenic growth factor. However, the role of FGF2 in atherosclerotic pathogenesis is far less understood [24, 25]. Our results confirmed that knockout of 18-kDa FGF2 in ApoE-/- mice possessed an inhibitory effect on the progression of atherosclerosis at different stages.

Macrophages have an essential role during all stages of atherosclerosis. When monocytes infiltrate the intima, they differentiate into macrophages and engulf lipoproteins into foam 
cells $[8,26]$. Apoptosis of endothelium cells, which cannot be cleared away from vessels immediately, would cause gradual accumulation of foam cells [27]. In a study by Tanaka et al [28]., FGF-2 treatment increased the number of vasa vasorum and accumulation of Mac3positive macrophages in atherosclerotic lesion formation, which is consistent with our results. In our study, we further confirmed that deletion of 18kDa FGF-2 was associated with reduced infiltration and accumulation of macrophages at different stages of atherosclerosis.

MCP-1 is considered a critical factor in the formation of atherosclerotic plaques, which has strong and specific chemotactic activity on monocytes. Enhanced expression of MCP1 was demonstrated in a variety of pathologic conditions, associated with inflammation and mononuclear cell infiltration. Extensive experimental evidence suggests that MCP-1 is highly expressed in atherosclerotic plaques, and mediates macrophage recruitment in the atherosclerosis lesion. In recent years, there is increasing evidence that MCP-1 plays a key role in the development of atherosclerosis [29-31]. Hypercholesterolemia induced higher expression of adhesion molecules such as VCAM-1 at atheroprone areas, which is also involved in transmigration, accumulation and differentiation of inflammatory cells into foam cells to increase intima-media thickness [32]. In the present study, we found that knockout of the 18-kDa FGF- 2 could reduce the expression of both MCP-1 and VCAM-1 in the plaque lesion. These findings indicate that deletion of 18 -kDa FGF- 2 contributes to reduce the inflammation in all of the stages of atherosclerosis process.

Oxidative stress plays a key role in the pathogenesis of atherosclerosis, particularly by promoting oxidative modification of low density lipoprotein (LDL). Oxidation of LDL is one of the earliest events in the formation of atherosclerosis, and it has been shown that NADPH oxidase is critically involved in the process in both mice and humans by acting either directly or indirectly as a precursor of ROS which are utilized as substrates by other enzymes to generate more powerful oxidizing species $[33,34]$. There are seven NADPH-dependent oxidoreductases (Nox enzymes) related isoforms, and four (Nox1, Nox2, Nox4, and Nox5) of them are expressed in vascular and immune cells $[31,35]$. Nox1 and 2 isoforms are involved in the development of atherosclerosis in mouse and human; both of them are activated by the subunit $\mathrm{p} 47 \mathrm{phox}$. Knockout of $\mathrm{p} 47 \mathrm{phox}$ in mouse protects against lesion formation in the aorta which suggests that either Nox1, Nox2 or both are important for the development of atherosclerosis [36]. Nox5 is upregulated in atherosclerotic lesions in human, but it is absent from the mouse genome; its impact on the development of lesions remains unknown.

Therefore, in our study, we chose p47phox to represent Nox1 and Nox2, and Nox4 which was comparatively obscure in atherosclerosis until recently. In order to evaluate the effect of the deletion of 18-kDa FGF-2 in the oxidative stress process in atherosclerosis, we tested the Nox4 and p47phox expression in the plaque. Compared to the ApoE-/- mice, 18-kDa FGF2 and ApoE-/- double knockout mice expressed lower levels of Nox4 and p47phox in aortic root sections.

In conclusion, our findings indicate that 18 -kDa FGF2, which previously was known only to be an angiogenesis factor, would aggravate the degree of atherosclerosis from early stage to advanced stage. Therefore, according to our current study, the consideration of using FGF2 as a therapeutic approache in cardiovascular disease should be avoided.

\section{Acknowledgements}

W.J.L conducted the experiments, analyzed data, H.M. Q.W and W.J.Y partially performed some experiments. J.J.Y designed and performed the experiments, analyzed data, wrote the manuscript, and convinced the whole project.

This work was supported by National Natural Science Foundation of China (Grant Numbers: 81400195, 81500339, 81500340).

\section{Disclosure Statement}

No potential conflicts of interest were disclosed. 


\section{Cellular Physiology Cell Physiol Biochem 2018;45:1434-1443 \begin{tabular}{l|l|l} 
DOI: 10.1159/000487569 & (c) 2018 The Author(s). Published by S. Karger AG, Basel
\end{tabular} and Biochemistry Published online: February 21, 2018 www.karger.com/cpb}

Liang et al.: Role of Low Molecular Weight FGF2 in Atherosclerosis

\section{References}

1 Krauss RM: Lipoprotein subfractions and cardiovascular disease risk. Curr Opin Lipidol 2010;21:305-311.

-2 Tertov VV, Orekhov AN, Kacharava AG, Sobenin IA, Perova NV, Smirnov VN: Low density lipoproteincontaining circulating immune complexes and coronary atherosclerosis. Exp Mol Pathol 1990;52:300-308.

3 Bobryshev YV, Ivanova EA, Chistiakov DA, Nikiforov NG, Orekhov AN: Macrophages and Their Role in Atherosclerosis: Pathophysiology and Transcriptome Analysis. Biomed Res Int 2016;2016:9582430.

4 Palmefors H, DuttaRoy S, Rundqvist B, Borjesson M: The effect of physical activity or exercise on key biomarkers in atherosclerosis--a systematic review. Atherosclerosis 2014;235:150-161.

5 Hansson GK, Libby P: The immune response in atherosclerosis: a double-edged sword. Nat Rev Immunol 2006;6:508-519.

6 Steffens S, Mach F: Inflammation and atherosclerosis. Herz 2004;29:741-748.

7 Moore KJ, Tabas I: Macrophages in the pathogenesis of atherosclerosis. Cell 2011;145:341-355.

-8 Moore KJ, Sheedy FJ, Fisher EA: Macrophages in atherosclerosis: a dynamic balance. Nat Rev Immunol 2013;13:709-721.

9 Carmeliet P: Mechanisms of angiogenesis and arteriogenesis. Nat Med 2000;6:389-395.

10 Folkman J, Klagsbrun M: Angiogenic factors. Science 1987;235:442-447.

11 Yanagisawa-Miwa A, Uchida Y, Nakamura F, Tomaru T, Kido H, Kamijo T, Sugimoto T, Kaji K, Utsuyama M, Kurashima C, et al.: Salvage of infarcted myocardium by angiogenic action of basic fibroblast growth factor. Science 1992;257:1401-1403.

12 Kawasuji M, Nagamine H, Ikeda M, Sakakibara N, Takemura H, Fujii S, Watanabe Y: Therapeutic angiogenesis with intramyocardial administration of basic fibroblast growth factor. Ann Thorac Surg 2000;69:1155-1161.

13 Post MJ, Laham R, Sellke FW, Simons M: Therapeutic angiogenesis in cardiology using protein formulations. Cardiovasc Res 2001;49:522-531.

-14 Simons M, Bonow RO, Chronos NA, Cohen DJ, Giordano FJ, Hammond HK, Laham RJ, Li W, Pike M, Sellke FW, Stegmann TJ, Udelson JE, Rosengart TK: Clinical trials in coronary angiogenesis: issues, problems, consensus: An expert panel summary. Circulation 2000;102:E73-86.

15 Liao S, Bodmer J, Pietras D, Azhar M, Doetschman T, Schultz Jel J: Biological functions of the low and high molecular weight protein isoforms of fibroblast growth factor-2 in cardiovascular development and disease. Dev Dyn 2009;238:249-264.

16 Uteza Y, Rouillot JS, Kobetz A, Marchant D, Pecqueur S, Arnaud E, Prats H, Honiger J, Dufier JL, Abitbol M, Neuner-Jehle M: Intravitreous transplantation of encapsulated fibroblasts secreting the human fibroblast growth factor 2 delays photoreceptor cell degeneration in Royal College of Surgeons rats. Proc Natl Acad Sci U S A 1999;96:3126-3131.

17 Liu MH1 TZ, Li GH, Qu SL, Zhang Y, Ren Z, Liu LS, Jiang ZS: Janus-like role of fibroblast growth factor 2 in arteriosclerotic coronary artery disease: atherogenesis and angiogenesis. Atherosclerosis 2013;229:10-17.

18 Seppo Yla-Herttuala BAL, Michael E. Rosenfeld: Expresssion of monocyte chemoattractant protein 1 in macrophage-rich areas of human and rabbit atherosclerotic lesions. Proc Natl Acad Sci U S A 1991;88:5252-5256.

-19 Yutaka Nakashima EWR, Andrew S. Plump: Upregulation of VCAM-1 and ICAM-1 at Atherosclerosis-Prone Sites on the Endothelium in the ApoE-Deficient Mouse. Arterioscler Thromb Vasc Biol 1998;18:842-851.

20 Bernard Lassegue KKG: NADPH Oxidases: Functions and Pathologies in the Vasculature. Arterioscler Thromb Vasc Biol 2010:653-661.

21 Jamel El-Benna PM-CD, Marie-Anne Gougerot-Pocidalo: p47phox, the phagocyte NADPH oxidase/Nox2 organizer: structure, phosphorylation and implication in diseases. Exp Mol Med 2009;41:217-225.

-22 Pakala R, Watanabe T, Benedict CR: Induction of endothelial cell proliferation by angiogenic factors released by activated monocytes. Cardiovasc Radiat Med 2002;3:95-101.

23 Slavin J: Fibroblast growth factors: at the heart of angiogenesis. Cell Biol Int 1995;19:431-444.

24 Chan J, Prado-Lourenco L, Khachigian LM, Bennett MR, Di Bartolo BA, Kavurma MM: TRAIL promotes VSMC proliferation and neointima formation in a FGF-2-, Sp1 phosphorylation-, and NFkappaB-dependent manner. Circ Res 2010;106:1061-1071.

25 Folkman J: Angiogenic therapy of the human heart. Circulation 1998;97:628-629. 


\section{Cellular Physiology Cell Physiol Biochem 2018;45:1434-1443 \begin{tabular}{ll|l|l|l|l} 
DOI: $10.1159 / 000487569$ \\
and Biochemistry 2018 The Author(s). Published by S. Karger AG, Basel
\end{tabular}}

Liang et al.: Role of Low Molecular Weight FGF2 in Atherosclerosis

-26 Wang Y, Wang GZ, Rabinovitch PS, Tabas I: Macrophage mitochondrial oxidative stress promotes atherosclerosis and nuclear factor-kappaB-mediated inflammation in macrophages. Circ Res 2014;114:421-433.

27 Tabas I, Garcia-Cardena G, Owens GK: Recent insights into the cellular biology of atherosclerosis. J Cell Biol 2015;209:13-22.

28 Tanaka K ND, Hirata Y: Augmented angiogenesis in adventitia promotes growth of atherosclerotic plaque in apolipoprotein E-deficient mice. Atherosclerosis 2011;215:366-373.

-29 Gerszten RE MF, Sauty A, Rosenzweig A, Luster AD: Chemokines, leukocytes, and atherosclerosis. J Lab Clin Med 2000;136:87-92.

30 Lin J, Kakkar V, Lu X: Impact of MCP-1 in atherosclerosis. Curr Pharm Des 2014;20:4580-4588.

-31 Weber C, Noels H: Atherosclerosis: current pathogenesis and therapeutic options. Nat Med 2011;17:14101422.

32 I. Masseau DKB: Carotid Endothelial VCAM-1 Is an Early Marker of Carotid Atherosclerosis and Predicts Coronary Artery Disease in Swine. J Biomed Sci Eng 2015;8:789-796.

-33 Jiang Q, Wang D, Han Y, Han Z, Zhong W, Wang C: Modulation of oxidized-LDL receptor-1 (LOX1) contributes to the antiatherosclerosis effect of oleanolic acid. Int J Biochem Cell Biol 2015;69:142-152.

-34 Yang JJ, Li P, Wang F, Liang WJ, Ma H, Chen Y, Ma ZM, Li QZ, Peng QS, Zhang Y, Wang SX: Activation of activator protein 2 alpha by aspirin alleviates atherosclerotic plaque growth and instability in vivo. Oncotarget 2016;7:52729-52739.

-35 Bedard K KK: The NOX family of ROS-generating NADPH oxidases: physiology and pathophysiology. Physiol Rev 2007;87:245-313.

-36 Barry-Lane PA PC, Van Der Merwe M: p47phox is required for atherosclerotic lesion progression in ApoE(/-) mice. . J Clin Invest 2001;108:1513-1522. 